Bio-grafía. Escritos sobre la Biología y su Enseñanza. ISSN 2027-1034

Número Extraordinario. p.p. 334-341

Memorias del Primer encuentro ambiental Universidad, ambiente y sustentabilidad: experiencias y prácticas.

\title{
SUSTENTABILIDAD COMO OPCIÓN DE VIDA: REFLEXIÓN EN TORNO AL RECURSO HÍDRICO
}

Rondón, D. ${ }^{1}$ Dávila, P., Espitia. V., Martínez, A. Mendoza. S., Mendoza. V. \& Pinzón, J. ${ }^{2}$

\section{Resumen}

Se vuelve necesario replantear la formación de los futuros actores políticos en el área de la investigación y en el área de las ciencias, generando espacios que permitan la integración de la formación disciplinar e investigativos, reconociendo la formación investigativa, como una de las posibilidades para el desarrollo de procesos de formación más consecuentes con el contexto social, político, económico y cultural, en el que se desenvuelve la educación en ciencia; por lo cual es de resaltar espacios de formación centrados en actividades de investigación, con el propósito de promover mayor experiencia, desde la articulación del interés mismo de los individuos con el trabajo investigativo partiendo del conocimiento adquirido en los espacios curriculares o extracurriculares, asumiendo la toma de decisiones y el control del propio aprendizaje, empezando a desempeñar el papel protagónico desde la acción investigativa (Dieleman \& Juarez, 2008). Con esto se puede redefinir el rol de "estudiantes pasivos" por el de "estudiantes investigadores" propendiendo por la formación de entes transformadores del cambio educativo-social, que estén en la capacidad de reconocer la importancia de la función de investigación.

Partiendo de lo anterior se espera un cambio de paradigma de transmisiónrecepción, convirtiendo ciudadanos en agentes activos, críticos y creadores del conocimiento, participando activamente en procesos de transformación social a través de la integración del conocimiento científico y la investigación, particularmente para este caso la importancia del recurso hídrico, como estrategia de abordaje y apropiación del conocimiento científico desde el

\footnotetext{
${ }^{1}$ Docente Líder Club de Ciencias Infundiendo Pensamiento Científico, drondon.quimica@gmail.com

${ }^{2}$ Estudiantes Club de Ciencias Infundiendo Pensamiento Científico
} 
Bio-grafía. Escritos sobre la Biología y su Enseñanza. ISSN 2027-1034

Número Extraordinario. p.p. 334-341

Memorias del Primer encuentro ambiental Universidad, ambiente y sustentabilidad: experiencias y prácticas.

contexto inmediato, reflexionando desde las dinámicas de consumo y hábitos en torno al manejo del agua.

Palabras Clave: Sustentabilidad, recurso hídrico, investigación, reflexión, conciencia ambiental.

\section{Abstract}

It becomes necessary to rethink the training of future political actors in the area of research and in the area of Science, creating spaces that allow the integration of disciplinary training and research, recognizing the research training as one of the possibilities for Process development Training More consistent with the social context, political, economic and cultural, in which education develops in science; so it is noteworthy areas: training focused on research activities for the purpose promoting mayor Experience from the articulation of Self Interest of Individuals with research work Based on the knowledge acquired in curricular or extracurricular spaces, assuming Decision-making and control of own Learning a Beginning play the leading role from the research Action (Dieleman \& Juarez, 2008). With this you can redefine the role of "Students liabilities" by "Research Students" Training prefering by entities Transformers educational and social change, they are in the ability to recognize the importance of the role of Research. Based on the above, it is expected the ONU 'paradigm shift transmission-reception, turning Citizens Assets, Critics and Creators of Knowledge Agents, actively participating in processes of social transformation, a Through Integration of Scientific Knowledge and Research , particularly paragraph This Case the Importance of water resources, as a strategy to approach and appropriation of Scientific Knowledge from the immediate context, reflecting from consumer dynamics and habits around Water Management.

Key Words: Sustainability, Water Resources, Investigation, Reflection, Environmental Awareness.

\section{INTRODUCCIÓN}

El desarrollo de un club de ciencias para la enseñanza de conceptos químicos, parte de la iniciativa de profesores en formación inicial, como una 
Bio-grafía. Escritos sobre la Biología y su Enseñanza. ISSN 2027-1034

Número Extraordinario. p.p. 334-341

Memorias del Primer encuentro ambiental Universidad, ambiente y sustentabilidad: experiencias y prácticas.

propuesta de abordaje conceptual y metodológico desarrollada con la finalidad de generar interés hacia el aprendizaje de la química, puesto que como futuros profesores de química desde los discursos y praxis que sustentan la formación inicial, se mantiene una tendencia marcada, en la cual las concepciones de química por parte de los estudiantes y docentes del área, se resumen en relación a una asignatura difícil y rigurosa, muchas veces atribuida a su relación con las matemáticas (Franco, Gallego, Pérez, Ramírez, Acosta, \& Rondón, 2014), que por lo general se encuentra desligada de los contextos, presenta carencia en el uso de materiales y problemáticas familiares, motivos por los cuales se presenta el desinterés por parte de los estudiantes.

Partiendo de lo anterior y desde los planteamientos de la investigación como estrategia pedagógica (IEP) de Mejía y Manjarrés (2012) dentro del espacio de club de ciencias se realizan aproximaciones investigativas como proyectos no curriculares en el área de ciencias; a partir de los cuales se pretenden aportar la construcción del conocimiento científico desde una perspectiva investigativa en el marco de la educación escolar, con el fin de propender por una mira desde la sustentabilidad, que permita la relación de elementos que caracterizan la dimensión y complejidad de la enseñanza de las ciencias en los distintos ambientes de formación, permitiendo la inclusión de aspectos de relevancia, en pro de generar y desarrollar procesos de construcción de ciudadanía critica, en relación con el contexto social, y cultural de las estudiantes. Esta propuesta surge como trabajo de investigación en la que se implementaron varios modelos y enfoques de investigación, desde la cual se pretende generar cambios a las metodologías que en ocasiones suelen ser propuestas de aula repetitivas, enmarcadas en los modelos de transmisión-recepción de contenidos curriculares las cuales están generando un retroceso metodológico; por lo que se propone un modelo de investigación- acción enmarcado en una pedagogía crítica propuesta Freire (1975) en el cual se pretende desarrollar procesos de innovación, en este caso del conocimiento científico, que ocasionen una amplia aceptación por parte de las estudiantes del Liceo Femenino Mercedes Nariño, siendo esta una estrategia novedosa e innovadora en donde el club de ciencias permitió la relación axiológica, social, científica y política entre las estudiantes y la naturaleza. Por esto como lo plantea Freire (1975) se necesita desarrollar la capacidad de ir más allá de los comportamientos esperados, partiendo de la curiosidad crítica del sujeto sin la cual se dificultan la invención y la reinvención de las cosas. Por lo cual se 
Bio-grafía. Escritos sobre la Biología y su Enseñanza. ISSN 2027-1034

Número Extraordinario. p.p. 334-341

Memorias del Primer encuentro ambiental Universidad, ambiente y sustentabilidad: experiencias y prácticas.

debe desafiar la capacidad creadora y a la curiosidad que nos caracterizan como seres humanos, y no atrofiarlas en el ejercicio de una mal llamada práctica educativa que en ocasiones suele inhibir estos aspectos. En este sentido, se considera que un proceso educativo acorde al contexto social es un proceso que tenga como finalidad sembrar en los individuos herramientas sociales que generen las habilidades de aprender a negociar, compartir, cuestionar, escuchar las diferentes opiniones de los seres humanos que los rodean en pro de generar una construcción del conocimiento durante cada día mediante la inclusión la escuela, desde la implementación de cuestiones socio científicas, como eje transversal contextualizado y dinamizador del conocimiento desde las problemáticas del recurso hídrico.

Partiendo de lo anterior y como lo define Rondón \& Gil (2015), desde la experiencia del Club de Ciencias Infundiendo Pensamiento Científico, se demuestra que la educación en ciencias no necesita centrarse en el aprendizaje pasivo e impreciso de conceptos, puesto que la enseñanza debería estar encaminada en la transformación de nuevos significados y percepciones de mundo, de tal manera que se logre la formación de sujetos creativos y críticos. De lo anterior se puede concebir una Educación en Ciencias para la Sustentabilidad (Sauvé, 2010), como la búsqueda del cambio constante, para mejorar las condiciones de un sistema socioecológico desde la escuela, donde el interés y la curiosidad de las estudiantes son el mayor recurso humano que se tiene para generar un accionar político, hacia las diferentes maquinarias e instituciones que tanto desfavorecen las condiciones actuales donde, en términos de Mejía y Manjarrés (2012), se vuelve necesario desarrollar la capacidad de ir más allá de los comportamientos esperados, partiendo de la curiosidad crítica del sujeto sin la cual se dificultan la invención y la reinvención de las cosas.

\section{OBJETIVO GENERAL}

Concientizar sobre las diversas problemáticas que se generan entorno al recurso hídrico.

\section{OBJETIVO ESPECÍFICO}

- Promover iniciativas que contribuyan a la concientización ambiental y el buen manejo de los recursos hídricos. 
Bio-grafía. Escritos sobre la Biología y su Enseñanza. ISSN 2027-1034

Número Extraordinario. p.p. 334-341

Memorias del Primer encuentro ambiental Universidad, ambiente y sustentabilidad: experiencias y prácticas.

TIEMPO DE DURACIÓN: 1 hora y 30 minutos

\section{MATERIALES}

1. Mesa de trabajo 1:

- Piedras

- Tierra

- Papel reciclado

- Agua sucia

- Tabla de porcentajes

\section{Mesa de Trabajo 2:}

- Fichas correspondientes a los materiales del filtro: Algodón, Gasa, Ceniza, Carbón activado, Arena y Piedras

- Ficha correspondiente a la información de cada material del filtro.

\section{METODOLOGÍA}

Para el correspondiente taller se proponen cuatro Ejes de abordaje, que se consideran adecuados para las diferentes dinámicas de trabajo enmarcadas en lo que se denomina Educación en Ciencias para la Sustentabilidad, como se presenta a continuación:

\section{Club de Ciencias como Espacio Dinamizador de la Enseñanza de Conceptos Científicos}

Rondón \& Gil (2015) plantean que profundizar en los intereses personales en unos tiempos determinados puede transformar no solo el conocimiento en el aula sino también en la comunidad, debido a que este tipo de espacios superan la rigidez del aula, debido a que desarrolla un entorno de diálogo de saberes gracias al intercambio de perspectivas y de experiencias que como lo plantea quien reflexiona sobre la acción humana y las relaciones que vive; por tal razón, es fundamental analizar desde este aspecto el desarrollo del trabajo y de los diferentes proyectos de investigación dentro del Club de Ciencias para que tengan una real incidencia el contexto de las estudiantes, su comunidad y la educación en general. 
Bio-grafía. Escritos sobre la Biología y su Enseñanza. ISSN 2027-1034

Número Extraordinario. p.p. 334-341

Memorias del Primer encuentro ambiental Universidad, ambiente y sustentabilidad: experiencias y prácticas.

\section{Investigación en la Escuela}

Este eje se propone teniendo en cuenta que al ser humano se le debe desafiar la capacidad creadora y la curiosidad que lo caracteriza, siendo estos dos aspectos fundamentales en el desarrollo de una investigación, esto se ve inhibido muchas veces por una mal llamada práctica educativa debido a que no es acorde al contexto social (Freire, 1975 citado en Manjarres \& Mejía 2012), por tal razón desde el Club de Ciencias se propone realizar proyectos investigativos que surjan del contexto mismo de las estudiantes, en este caso con el fin de que se propongan soluciones a problemáticas alrededor del consumo del recurso hídrico

\section{DESCRIPCIÓN DE LAS ACTIVIDADES}

Mesa de trabajo 1: Una persona en la postura de un ciudadano del "común" envía el agua a cualquier sector productivo después de consumir (Minería, Hidroeléctricas, Agricultura, Ganadería, construcción, Educación), posterior a esto las industrias argumentan que uso le darán al agua y luego a que sector enviarla, hasta llegar al educador ambiental, que será obligatoriamente el penúltimo y este la devolverá de nuevo al ciudadano del común (existirán costos que varían en sentido a si deciden descontaminar o seguir contaminando el agua)

Mesa de Trabajo 2: Construcción simbólica de un filtro o purificador para descontaminar el agua de acuerdo a la función de cada material que compone el filtro; los partícipes deben llegar a un acuerdo que permita la utilización del recurso hídrico para un bien común.

\section{REFLEXIONES}

Dentro del espacio de club de ciencias y partiendo de la reflexión propuesta en el desarrollo metodológico, se plantea que el cuidado de ríos, quebradas, manantiales y cualquier otro tipo de fuente hídrica son necesarios e importantes, ya que es el principal proveedor del agua para todos. Parece que esto se ha olvidado y la mano del hombre ha hecho lo contrario, convirtiendo las fuentes hídricas en el 'carrasco' de muchas comunidades, lo cual ya pasa 'su cuenta de cobro' y con cada llegada del invierno se ven las consecuencias, que han terminado en hechos lamentables como inundaciones, sequías y cambios de clima, afectando pueblos, ciudades y la 
Bio-grafía. Escritos sobre la Biología y su Enseñanza. ISSN 2027-1034

Número Extraordinario. p.p. 334-341

Memorias del Primer encuentro ambiental Universidad, ambiente y sustentabilidad: experiencias y prácticas.

vida de cientos de personas. Es momento de hacer un alto, reflexionar y efectuar un cambio que incluya mayor concientización ambiental siguiendo a la sustentabilidad como opción de vida donde tendremos siempre el equilibrio que debemos mantener para vivir conscientemente y no destruir nada basándonos en cosas "pequeñas" con materiales básicos con los cuales podemos mejorar la vida.

De lo anterior y desde lo que plantea Rondón \& Gil en 2015, el agua potable es muy reducida a comparación del agua no apta para el consumo, es importante tomar conciencia de ahorro y limpieza de la misma; según el Ministerio de Ambiente, Vivienda y Desarrollo un 95\% de aguas Residuales Domésticas se vierten sin tratamiento alguno, $85 \%$ de las Aguas Residuales Industriales se vierten sin tratamiento adecuado y el $95 \%$ de aguas residuales agrícolas se vierten sin tratamiento. Debido a lo anterior una óptima calidad de vida no solo depende de la decodificación del ambiente en la vida cotidiana, sino que, al contrario, la falencia que se presenta en la sociedad actual refiere a la apropiación del territorio debido a la dificultad de una "educación ambiental" en el contexto colombiano. Al respecto, Sauvé (2010) propone que la educación debe ir de la mano con la sustentabilidad para lograr una conexión armónica entre un sistema político, económico, tecnológico, científico, social y cultural en pro de la preservación del ambiente, por tal razón, este eje se plantea en el desarrollo de la propuesta para analizar una verdadera incidencia en las estudiantes acerca de su relación con el ambiente, siendo esto fundamental en la apropiación de una propuesta de Educación en Ciencias para la Sustentabilidad.

\section{BIBLIOGRAFÍA}

- Comisión Distrital de Ciencia, T. e. (OCTUBRE de 2007). PLAN DE CIENCIA, TECNOLOGÍA E INNOVACIÓN BOGOTÁ D.C. 2007-2019 "BOGOTA SOCIEDAD DEL CONOCIMIENTO". Obtenido de PLAN DE CIENCIA, TECNOLOGÍA E INNOVACIÓN BOGOTÁ D.C. 20072019 "BOGOTA SOCIEDAD DEL CONOCIMIENTO": www.desarrolloeconomico.gov.co/4- normatividad plan ciencia y tecnología 
Bio-grafía. Escritos sobre la Biología y su Enseñanza. ISSN 2027-1034

Número Extraordinario. p.p. 334-341

Memorias del Primer encuentro ambiental Universidad, ambiente y sustentabilidad: experiencias y prácticas.

- Dieleman, H., \& Juárez, M. (abril de 2008). ¿CÓMO SE PUEDE DISEÑAR EDUCACIÓN PARA LA SUSTENTABILIDAD? Revista Internacional Contaminación Ambiental, 3(24), 131-147.

- Gallopín, G. (mayo de 2003). Sostenibilidad y desarrollo Sostenible: Un enfoque sistemático. CEPAL-SERIE Medio ambiente y desarrollo, 1-46. Chile.

- Hernández Romero, L. (2010). Los proyectos de aula y el aprendizaje por investigación. Boletín PPDQ, 17-21.

- Mejía, M. R., \& Manjarrés, M. E. (2012). La investigación como Estrategia Pedagógica: Una construcción de cultura ciudadana y democrática desde el Sur. (E. A. Española, Ed.) Colombia: Académica Española

- Pierri, N. (2005). Historia del concepto de desarrollo sustentable. En G. Foladori, \& P. Naína, ¿Sustentabilidad? Desacuerdos sobre el desarrollo sustentable (págs. 27-81). México.

- Rondón, D. \&. (2015). Educación en Ciencias para la Sustentabilidad en la Escuela: Una propuesta para la enseñanza de la Química desde el Club de Ciencias. X Convención sobre Desarrollo Sostenible y Educación Ambiental. Habana, Cuba: Ministerio de Ambiente y Tecnología de Cuba.

Sauvé, L. (2010). EDUCACIÓN CIENTÍFICA Y EDUCACIÓN AMBIENTAL: UN CRUCE FECUNDO. ENSEÑANZA DE LAS CIENCIAS, 28(1), 518.

UNESCO. (2006). Decenio de las Naciones Unidas de la Educación con miras al Desarrollo Sos. Plan de aplicación internacional, Organización de las Naciones Unidas para la Educación, la Ciencia y la Cultura. Recuperado el 26 de agosto de 2014. 